ARTIGO ORIGINAL ORIGINAL ARTICLE

\section{Regulação dos medicamentos inovadores em Portugal enquanto exemplo de transparência, accountability e governança pública: nem todas as áreas nascem iguais}

\author{
Regulation of innovative drugs in Portugal as an \\ example of transparency, accountability and public \\ governance: not all areas are born equal
}

Pedro Miguel Alves Ribeiro Correia', Maria João Vergueiro²

DOI: $10.21115 / J B E S . v 10 . n 2 . p 140-147$

\section{RESUMO}

Objetivo: A regulação como uma área da governança, está patente nas diversas dimensões da administração pública atual. A atividade de regulação das tecnologias da saúde em Portugal é executada pelo INFARMED (Autoridade Nacional do Medicamento e Produtos de Saúde), enquanto entidade autónoma administrativa sob a tutela do Ministério da Saúde, exercendo um papel fundamental na avaliação do financiamento dos medicamentos inovadores pelo Estado. Foi criada uma figura designada de Autorização de Utilização Especial (AUE) que permite acesso a medicamentos que estão sob processo de avaliação pelo INFARMED, ainda não comparticipados, o que representa um esforço financeiro extra por parte do Estado. Neste trabalho os autores pretendem analisar as AUEs concedidas num determinado período de tempo com o propósito de identificar áreas prioritárias de estratégia administrativa e de regulação. Métodos: Foram utilizados neste trabalho dados do INFARMED referentes às AUE concedidas no período de 1 de janeiro a 31 de agosto de 2016. A análise encontra-se subdividida por categorias de análise: frequência absoluta de cada área terapêutica e frequência absoluta de cada medicamento. Resultados: Da análise dos dados ressaltam duas realidades, $60 \%$ das AUE concedidas são referentes à área terapêutica da oncologia, sendo a segunda área terapêutica mais visada a oftalmologia que corresponde a apenas 15\%; e dos 10 fármacos mais requisitados por AUE, 7 são utilizados para tratamento de doenças oncológicas. Conclusões: Desta análise se infere que os esforços das entidades administrativas e reguladoras e as reformas políticas no que toca ao medicamento devem incidir sobretudo na área da patologia oncológica, tendo em conta o actual panorama crescente no que toca às tecnologias de inovação na saúde.

\section{Keywords:}

public administration, INFARMED, regulation, innovative therapies, public policies

\section{ABSTRACT}

Objective: Regulation as an area of governance becomes evident in the different dimensions of contemporary public administration. The regulatory activity concerning health technologies in Portugal is carried out by INFARMED (National Authority of Medication and Health Products), as an autonomous administrative entity under the Ministry of Health's jurisdiction, playing a fundamental role in the evaluation of the innovative drugs' financing terms by the State. A figure called Special Authorization Use (AUE) was created to allow access to drugs that are under evaluation by INFARMED, which are not yet reimbursable, representing a significant financial effort by the State. In this work the authors analyzed AUEs granted during a determined period of time in order to identify

\footnotetext{
Recebido em: 23/06/2016. Aprovado para publicação em: 17/03/2018.

1. Doutoramento em Ciências Sociais (Especialidade em Administração Pública) - Universidade Técnica de Lisboa (UTL); Licenciatura em Estatística e Gestão de Informação - Universidade Nova de Lisboa (NOVA); Professor do Insituto Superior de Ciências Sociais e Polítcas (ISCSP) - Universidade de Lisboa (ULisboa); Investigador Integrado do Centro de Administração e Políticas Públicas (CAPP) - ISCSP-ULisboa; Investigador Colaborador do Centro Interdisciplinar de Estudos de Género (CIEG) - ISCSP-ULisboa; Consultor da Direção-Geral da Política de Justiça (DGPJ), Ministério da Justiça; Coordenador do Observatório Nacional de Administração Pública (ONAP), ISCSP-ULisboa.

2. Doutoranda em Administração da Saúde, Instituto Superior de Ciências Sociais e Políticas (ISCSP), Universidade de Lisboa (ULisboa); Licenciatura em Medicina pelo Instituto de Ciências Biomédicas Abel Salazar, Universidade do Porto (ICBAS-UP); Assistente Hospitalar de Oncologia Médica; Therapeutic Area Head Solid Tumors - Medical Department, na Novartis Oncology Portugal.
} 
critical areas for administrative and regulatory strategies. Methods: The authors used data from the INFARMED for AUEs granted from January 1st to the 31st of August 2016. This analysis is divided according to category: absolute frequency of each therapeutic area and absolute frequency of each drug. Results: Data analysis highlights two realities: $60 \%$ of the AUEs granted refer to the oncology therapeutic area, being the second most frequent the ophthalmologic area corresponding to only 15\%; and 7 out of the 10 most required drugs through AUEs are used to treat oncological diseases. Conclusions: From this analysis, it can be inferred that the efforts of the administrative and regulatory authorities and the political reforms regarding drug policies should focus mainly on the area of cancer pathology, considering the current growing panorama regarding the technologies of innovation in health.

\section{Introdução}

A governança representa um paradigma de reforma da administração pública contemporânea alvo de intensa investigação e menção na literatura e no ambiente académico atual. No entanto, alguns dos conceitos que a palavra encerra são tão antigos como a história da humanidade (Emmerij, Jolly, \& Weiss, 2001).

A admissão ao léxico internacional da administração e das políticas públicas, da Governança, deu-se nas décadas de 80 e 90 do século XX, permeando de uma forma quase universal a agenda e o discurso das atividades financiadas por instituições públicas e privadas (Weiss, 2000). A adoção de princípios de boa governança por parte das instituições financeiras mundiais como critério de seleção dos países ou entidades passiveis de receberem ajuda financeira externa foi um ponto fulcral nesta adoção universal da teoria administrativa que o termo encerra. As medidas a implementar nos países, exigidas pelas grandes instituições financeiras, como o Fundo Monetário Internacional e o Banco Mundial, em contrapartida aos programas de ajuda financeira, tinham como objetivo assegurar a disciplina fiscal e monetária, mas também encerravam em si uma exigência a longo prazo de restruturação do papel do Estado na alocação dos recursos do mercado (Nanda, 2006).

O grande impulso na divulgação da governança enquanto teoria da administração pública nos círculos académicos foi dado sobretudo com a contribuição dos autores Laurence E. Lynn Jr., Carolyn J. Heinrich, e Carolyn J. Hill (Lynn \& Heinrich, 2000; Lynn, Heinrich \& Hill, 1999, 2001) cujo trabalho representa uma discussão alargada deste campo de investigação, das questões e desafios que, do ponto de vista de políticas e de administração pública, levanta essa mesma investigação, e das estruturas institucionais necessárias para se encontrarem soluções para os problemas administrativos. Os autores sugerem que a governança como teoria administrativa tem o potencial de unificar a literatura sobre políticas públicas e administração pública que se começava a espalhar no início dos anos 80 e 90 do século passado, aplicando-Ihe objetivos e interpretações comuns e destacando uma contribuição crítica de um conjunto robusto de investigação que se desenvolveu nessa área (Frederickson et al., 2012). Lynn e os seus colegas defendem que a grande questão no cerne da pesquisa científica relacionada com a governança é: "como podem os regimes, agências, programas e atividades do sector público ser organizados e geridos para atingirem os propósitos públicos?" (Lynn et al., 2001).

Mas o que significa exatamente governança e boa governança? De que forma estão tão enraizadas no discurso político e administrativo se encontramos na literatura diversas definições do termo, nem sempre totalmente concordantes?

Foi Harlan Cleveland nos anos 70 que primeiro utilizou a palavra "governança" como uma alternativa à expressão "administração pública", defendendo-a nos seus múltiplos discursos e publicações como a corrente de orientação administrativa mais próxima dos indivíduos e mais capaz de enquadrar as soluções para as questões da administração pública contemporânea (Cleveland, 1972).

A "Comissão da Governança Global" descreve governança como o conjunto das diferentes formas que os indivíduos e as instituições públicas e privadas utilizam para gerirem os seus assuntos comuns, sendo um processo contínuo no qual os interesses comuns e também os divergentes podem ser acomodados em função de ações cooperativas que possam ser tomadas (Commission on Global Governance, 1995).

Os autores Lynn, Heinrich e Hill (Lynn et al., 2001) descrevem este fenómeno de adoção dos princípios da governança na esfera pública e privada como a forma de enquadrar as decisões e ações globais e locais e como referência às normas formais e informais de determinada instituição, não sendo obrigatória uma definição formal, já que na verdade o termo apresenta uma componente intuitiva tão intensa que quem o aplica está enquadrado de uma forma automática. Nesta perspetiva, quando os autores utilizam o termo governança como uma forma de atingir objetivos políticos ou organizacionais pode não ser claro se se referem à estrutura organizacional, a processos administrativos, a incentivos e regras, a filosofias administrativas, ou a uma combinação destes e de outros elementos.

De acordo com Frederickson (Frederickson et al., 2012) existem cinco problemas principais, na governança, que podem predizer entraves à sua correta aplicação na prática. Um deles é tratar-se de um conceito administrativo popular e considerado "na moda" com tudo o que de positivo (in- 
teresse académico, grande volume de produção científica) e de negativo (efemeridade, perda de qualidade na investigação disseminada) acarreta, sendo um segundo problema relacionado com este, o facto de ser impreciso, podendo tornar as pesquisas e a investigação mais complexas e dando ainda espaço para diferentes interpretações aquando das tentativas de implementar na prática o defendido na academia. A imprecisão na definição acarreta ainda o problema da conceção de valores, que está implícita ao tema, poder ter diferentes interpretações, podendo não ser percecionados da mesma forma universalmente e gerar uma concordância falsa entre académicos ou até mesmo entre burocratas. Em terceiro lugar o termo governança carrega uma associação a visões negativas pré-existentes acerca das instituições governamentais e burocráticas. Depois é sobretudo sobre mudança prospetiva e sobre implementação de reformas, não passando necessariamente por uma descrição das relações interjuridiscionais entre os sectores público e privado. Por último, a governança tende a dar uma ênfase e importância desmesuradas às instituições não-governamentais, ou seja, raramente os serviços prestados pelas instituições públicas são desprovidos de uma relação ou parceria público-privada.

Citando Janet Newman, é interessante notar que a governança falha em lidar adequadamente com questões de diversidade e padrões de inclusão nos quais é baseada (Newman, 2000).

Após análise de algumas destas diferentes abordagens à governança sobressai o facto de não parecer que este conceito traga realmente algo totalmente novo à administração pública, mas em todos os casos parece existir o ponto comum da necessidade da envolvência de diversos parceiros, públicos e privados, na tentativa de garantir o melhor, mais justo e equitativo serviço público possível.

Os académicos dedicados à governança devem estar preparados para explicar não só o que é a governança, mas também o que esta não é (Frederickson, 2009).

\section{Regulação no contexto da governança}

O interesse académico na regulação como forma de governança ou como fazendo parte dela, e no estado regulador como uma das suas partes mais características, sofreu um aumento marcado nas últimas décadas.

Desde meados dos anos 80 do século XX que a governança através da regulação deixou de ser uma peculiaridade da administração pública americana para passar a ser central nas reformas na União Europeia (Majone, 1994), América Latina (Jordana \& Levi-Faur, 2003; Manzetti, 2000), Ásia (Jayasuriya, 2001) e na generalidade dos países em vias de desenvolvimento (Cook et al., 2005).

A regulação como um dos pontos-chave da governança, como uma realidade institucional, como um campo de estudo académico e como um discurso público ganhou um espaço de destaque na esfera da vida política, patente no avanço institucional da regulação no contexto da privatização e do neoliberalismo, com a criação de mais e mais entidades reguladoras autónomas, no desenvolvimento de políticas para a promoção da competitividade económica (regulation-for-competition), estando as novas autoridades regulatórias empenhadas em atingir e promover a competição (more rules, more competition) (Vogel, 1998).

A transferência do conhecimento de regulação da esfera unicamente política para um contexto mais social faz sentido à luz dos princípios da governança na medida em que as entidades regulatórias têm uma influência clara sobre os ministérios podendo servir de alavancas na relação entre o estado e os interesses económicos, não esquecendo a parte social que deverá, em última análise, estar sempre patente na formulação das soluções do Estado.

Por outro lado, os benefícios e custos que a regulação impõe aos negócios e, em particular, às entidades privadas envolvidas em determinada situação não são claros e têm também de ser acautelados no sentido de promover a sustentabilidade económica do país (Jordana \& Levi-Faur, 2004).

As diversas definições de regulação refletem diferentes preocupações académicas, métodos de pesquisa, diferentes experiências pessoais, nacionais e históricas do formulador da definição. Baldiwn (Baldwin et al., 1998) identificou na literatura três significados principais:

- Regulação como uma forma específica de governança: um conjunto de regras autoritárias acompanhadas muitas vezes por uma agência administrativa para monitorizar e reforçar a compliance;

- Regulação como governança no sentido geral: esforço agregado de agências do Estado para guiar a economia;

- Regulação no seu sentido mais lato: todos os mecanismos de controlo social, incluindo nãointencionais e processos não atribuíveis ao Estado.

A última definição tem ganho maior ênfase na literatura construtivista e socio-legal, muito derivado do aumento crescente de regimes internacionais semi-consensuais para a governança de problemas globais como armas de destruição massiva e as alterações climáticas (Jordana \& Levi-Faur, 2004).

As relações entre regulação e competição também sofreram mudanças nas últimas décadas. No início da década de 1970 George Stigler escreveu que "regulação e competição são amigos retóricos e inimigos mortais" (Stigler, 1975).

Apesar desta noção da relação entre regulação e competição ser ainda parte do discurso público e político, poderá já não refletir a interação contemporânea entre regulação e competição. A "regulation-for-competition" que refina o trabalho dos reguladores representa um avanço tecnológico profissional para a regulação. A par das novas tecnologias 
digitais e da melhoria da qualidade técnica humana das agências reguladoras assiste-se ao aparecimento de mercados mais livres ao mesmo tempo que se consolidam os regimes regulatórios sobre estes. Esta nova realidade permite aos reguladores um alinhamento entre eles e com os parceiros sobre os quais exercem a sua atividade regulatória que permite uma maior legitimidade da relação e um resultado mais coerente com os interesses públicos.

\section{Contexto normativo português da regulação do medicamento}

A Autoridade Nacional do Medicamento e Produtos de Saúde, I. P. (INFARMED), é um organismo sob a tutela do Ministro da Saúde de Portugal, dotado de autonomia administrativa, financeira e património próprio, e integrado na administração indireta do Estado, cuja principal função é a regulação dos sectores dos medicamentos de uso humano, dispositivos médicos e produtos cosméticos (Ministério da Saúde, 2012).

No âmbito da sua atividade reguladora o INFARMED exerce funções no processo de comparticipação e financiamento dos medicamentos por parte do Estado, o que requer uma detalhada avaliação farmacoterapêutica mas também farmocoeconómica de forma a garantir a racionalidade na comparticipação e aquisição dos mesmos e uma distribuição eficiente dos recursos finitos do Estado.

Esta função de avaliação de tecnologias de saúde, que ocorre em Portugal para os medicamentos, desde 1999, no âmbito dos processos de comparticipação ou de avaliação prévia antes da decisão de financiamento, viu a sua abrangência alargada em 2015 com a criação do SiNATS - Sistema Nacional de Avaliação de Tecnologias de Saúde.

O SiNATS surgiu tendo em consideração a crescente evolução no que toca à inovação e ao desenvolvimento de novas tecnologias na área da saúde, além dos medicamentos, como é o caso dos dispositivos médicos, sendo o seu principal objetivo garantir uma avaliação sustentada da contribuição de determinada inovação para os ganhos em saúde (Ministério da Saúde, 2015).

A criação do SiNATS veio ainda possibilitar a introdução de medidas que garantem a transparência, a previsibilidade, a equidade na utilização e a obtenção de ganhos em saúde que justifiquem o financiamento público. A avaliação do valor das tecnologias financiadas pelo Estado é realizada de uma forma contínua, ao longo do seu ciclo de vida, o que permite aumentar a garantia da eficiência da decisão do financiamento. Além disso pretende-se também garantir o envolvimento de Portugal nos exercícios comunitários de criação de um sistema integrado e europeu de avaliação de tecnologias de saúde.

A CATS - Comissão de Avaliação de Tecnologias de Saúde - foi criada no âmbito do SiNATS, como a comissão respon- sável pela emissão dos pareceres e recomendações, apreciação de estudos de avaliação económica e como proponente de medidas adequadas aos interesses de saúde pública e do Serviço Nacional de Saúde relativamente a tecnologias de saúde (INFARMED, 2016). À CATS compete, de forma genérica, emitir pareceres e recomendações, apreciar o valor acrescentado das tecnologias e os estudos de avaliação económica. A CATS é composta por um conjunto de personalidades com qualificações, experiência e formação especializada, nomeadamente nas áreas das ciências médicas, farmacêuticas, económicas e estatísticas.

Os pareceres do SiNATS, emitidos após avaliação baseada no conhecimento técnico do INFARMED e em todo o conjunto de informações organizadas e estruturadas pela CATS, com vista a proceder a essa mesma avaliação, podem resultar em recomendações gerais para a utilização de determinada tecnologia da saúde no Sistema Nacional de Saúde (SNS), bem como em condições de utilização e aquisição pelas instituições e serviços do Serviço Nacional de Saúde. Cabe ao titular da Autorização de Introdução no Mercado (AIM) do medicamento o ónus da prova quanto à eficácia, ao valor terapêutico acrescentado ou à sua equivalência terapêutica e à sua vantagem económica.

Há claramente necessidade de comprovar a qualidade, segurança e eficácia de determinada tecnologia de saúde para que esta possa ser considerada como passível de ser introduzida no mercado. No entanto, apesar de condições necessárias, não são as únicas a garantir o seu financiamento pelo SNS. O controlo da eficiência e efetividade que permita demonstrar que os recursos públicos destinados à prestação de cuidados de saúde são utilizados em tecnologias de saúde que oferecem mais-valias relevantes, é um dos principais propósitos da criação do SiNATS.

Com o SiNATS surge a introdução do contrato como forma de regulação das relações do Estado com os titulares das AIM e de utilização dos medicamentos, dispositivos ou outras tecnologias de saúde, com o propósito de estabelecer uma corresponsabilização no entendimento do financiamento, mas também para garantir uma participação activa das entidades privadas na definição da situação jurídica de cada uma das tecnologias de saúde.

Os contratos enquadram as condições de comparticipação ou da decisão de aquisição mediante avaliação prévia da tecnologia de saúde em questão e definem o compromisso do titular dessas tecnologias de acordo com os objetivos do sistema de saúde.

Os medicamentos que se encontram em avaliação prévia pelo SiNATS não podem ser adquiridos pelas entidades tuteladas pelo Ministério da Saúde sem que tenha sido celebrado o contrato, salvo em caso excecionais, autorizados pelo conselho diretivo do INFARMED mediante requerimento fundamentado da instituição ou serviço, nomeadamente 
quando se verifique a ausência de alternativa terapêutica ou em casos em que o doente corra risco imediato de vida ou de sofrer complicações graves - Autorização de Utilização Especial (AUE) (Ministério da Saúde, 2006).

As autorizações concedidas ao abrigo desta figura têm sempre carácter temporário e meramente transitório, cessando sempre que o medicamento passe a estar efetivamente comercializado em Portugal.

De uma forma sucinta, para que determinado medicamento seja dispensado ao abrigo de AUE é necessário reunir determinadas condições, nomeadamente, uma descrição fundamentada do clínico que pretende administrar o medicamento, baseada em evidência científica que suporta a sua decisão clínica, perante a ausência de uma alternativa no mercado. Este pedido passa por uma primeira avaliação técnico-económica pela comissão de farmácia e terapêutica e pelo conselho de administração da instituição do SNS ao qual pertence o clínico, sendo que, no caso de ser validada é enviada para avaliação pelo Conselho Diretivo do INFARMED. Caso a deliberação final seja positiva, a instituição tem o aval para adquirir o medicamento junto da empresa detentora da AIM do mesmo para que possa ser dispensado ao doente. Esta aquisição é realizada sem qualquer tipo de negociação, dado tratar-se de uma situação excecional, ficando o encargo financeiro à custa do orçamento da própria instituição que o requisita.

É sabido empiricamente que estas situações ditas especiais enquadram, grande parte das vezes, medicamentos inovadores que se encontram em processo de avaliação farmocoeconómica e terapêutica pelo INFARMED. Também de forma empírica é descrito por agências noticiosas e nos fóruns de discussão sobre inovação terapêutica que a área da patologia oncológica é a responsável por um grande número destas autorizações especiais. Este fenómeno tem duas explicações possíveis e muitas vezes convergentes: a área da patologia oncológica é uma das que maior investimento tem recebido por parte da indústria farmacêutica (EFPIA, 2014) e o processo negocial entre a autoridade e o detentor da AIM tende a ser mais moroso nos processos referentes a fármacos de elevado custo para o Estado, como é o caso dos medicamentos inovadores.

No atual contexto de rápido aumento do número de tecnologias inovadoras em saúde, que poderão trazer verdadeiros ganhos em saúde, mas que acarretam necessariamente um aumento do custo direto e indireto para o Estado, é evidente a necessidade de mecanismos e entidades reguladoras eficientes, com respostas e negociações em tempo útil, de forma a garantir a alocação e a utilização dos recursos económicos do país em prol dos cidadãos.

Neste trabalho pretende-se enquadrar a realidade no que respeita às AUE de medicamentos concedidas em Portugal à luz de uma figura criada para uso em situações excecionais, mas que poderá estar a tornar-se, tendo em conta a crescente importância da inovação terapêutica no seu contexto clínico e económico, uma regra com o consequente impacto orçamental para o Estado.

\section{Metodologia}

Para o presente trabalho foram utilizados dados do INFARMED relativos à concessão de AUE no SNS em Portugal, no período compreendido entre 1 de janeiro e 31 de agosto de 2016.

A análise encontra-se subdividida por categorias de análise: frequência absoluta de cada área terapêutica e frequência absoluta de cada medicamento.

No período analisado o INFARMED concedeu um total de 1229 AUE relativas a 83 medicamentos distintos originários de 19 áreas terapêuticas diferentes. Como tal, é possível considerar a análise empírica como extremamente robusta.

\section{Resultados}

A Figura 1 apresenta a frequência absoluta das AUE por área terapêutica para o período compreendido entre 1 de janeiro e 31 de agosto de 2016, de acordo com os dados do INFARMED (em escala logarítmica).

É notório que os dados são bem aproximados por uma lei de potências com expoente -1,899, o que fica espelhado pelo coeficiente de determinação extremamente elevado $\left(R^{2}=0,9517\right)$. Este facto tem como implicação que a maior parte das AUE advêm de um pequeno número de áreas terapêuticas, o que, é possível argumentar, poderá ter impactos importantes ao nível das características da gestão e da governança na área da saúde e, em particular, das políticas do medicamento.

Realce-se que, das 19 áreas terapêuticas registadas pelo INFARMED no período em análise, 4 destas1 (correspondendo apenas a cerca de $21,1 \%$ do total) contabilizam 1.043 entradas (correspondendo a uns incontornáveis $84,9 \%$ do total).

De notar que se conhecem outras áreas da administração pública onde se verifica este tipo de comportamento, como é o caso da justiça (Correia \& Jesus, 2016).

A figura 2 apresenta, a frequência absoluta de cada medicamento para o período compreendido entre 1 de janeiro e 31 de agosto de 2016, de acordo com os dados do INFARMED (em escala logarítmica).

Neste particular, é notório que os dados são bem aproximados por uma função exponencial, o que fica espelhado no coeficiente de determinação extremamente elevado $(R 2=0,9716)$. Este facto tem como implicação que a maior parte das autorizações especiais dizem respeito a um pequeno número de medicamentos, o que, é possível argumen-

1 Oncologia, oftalmologia, pneumologia e hepatologia. 


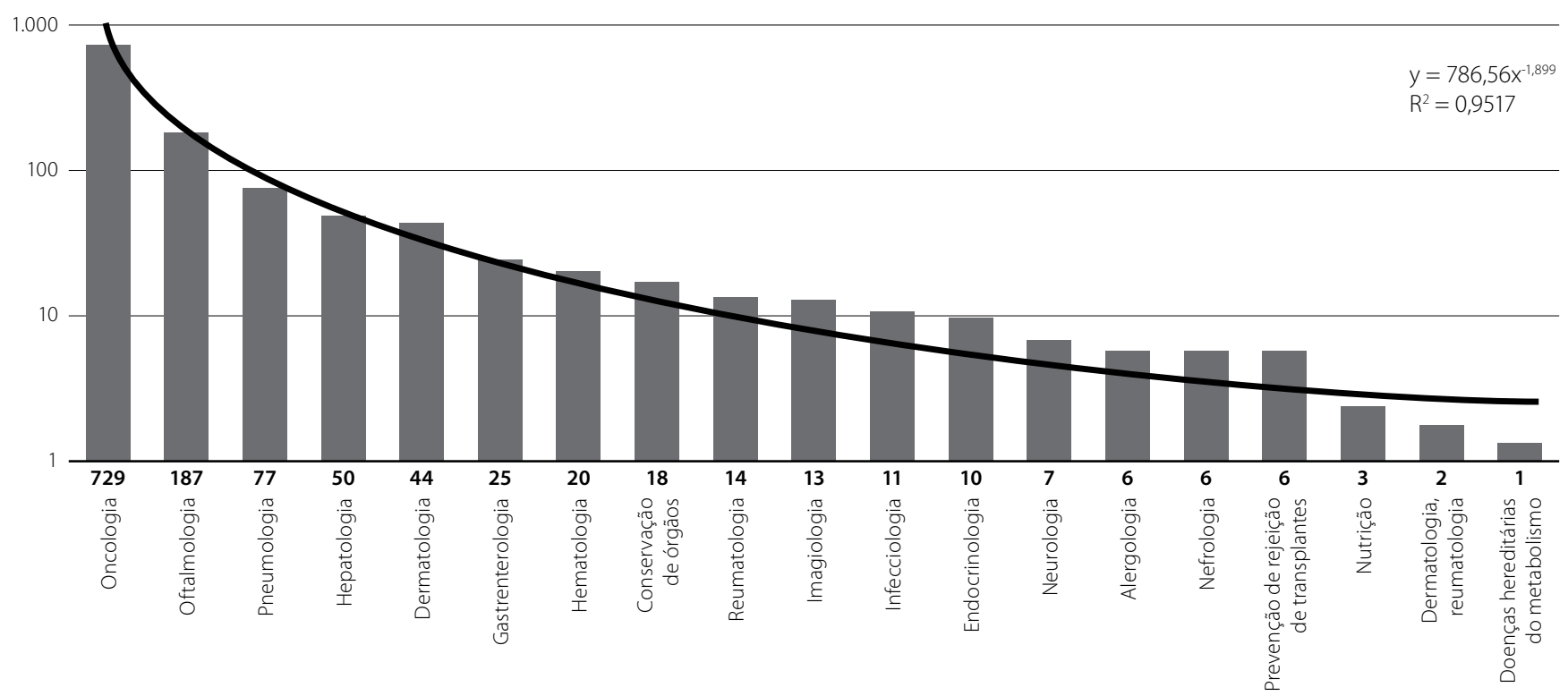

Fonte: elaboração própria com base em dados do INFARMED.

Figura 1. Frequência absoluta das AUE por área terapêutica, 1 de janeiro de 2016 a 31 de agosto de 2016 (escala logarítmica).

tar, poderá ter, de forma análoga ao verificado para as áreas terapêuticas de origem das autorizações especiais, impactos importantes ao nível das características da gestão e da governança na área da saúde e, em particular, das políticas do medicamento.

Realce-se que, dos 83 medicamentos autorizados por AUE no período em análise, 16 destes2 (correspondendo apenas a cerca de 19,3\% do total) contabilizam 710 entradas (correspondendo a uns expressivos 57,8\% do total).

Note-se, no entanto, que o fenómeno patente na Figura 2 obedece a uma lei exponencial que é menos intensa que a lei de potências que governa o fenómeno patente na Figura 1.

\section{Conclusões}

Com a recolha de informação do INFARMED foi possível determinar que a área terapêutica da oncologia foi, com uma margem de diferença bastante significativa para a segunda área terapêutica mais visada, aquela à qual um maior número de AUE foi concedida.

No total das 1229 AUE concedidas em instituições do Sistema Nacional de Saúde de Portugal, 729, que correspondem a cerca de $60 \%$ do total, eram referentes a fármacos utilizados no tratamento de doenças oncológicas. A oftalmologia foi a segunda área terapêutica com maior número de AUE concedidas, correspondendo a apenas 15\%, com um número absoluto de 187.

2 Aflibercept, Nivolumab, Pertuzumab, Trastuzumab Emtansina, Ranibizumab, Pemetrexedo, Idelalisib, Cobimetinib, Bevacizumab, Omalizumab, Paclitaxel, Daclatasvir, Nintedanib, Ibrutinib, Pirfenidona e Vedolizumab.
Dos 10 fármacos mais frequentemente requisitados em regime de AUE, 7 são da área da oncologia, e os restantes 3 da área da oftalmologia.

Em conjunto estas duas áreas terapêuticas contabilizam $74 \%$ do total das AUE no período considerado.

Perante a evidência descrita são estas duas áreas, e em particular a área terapêutica da oncologia, o alvo do maior número de AUE concedidas pelo INFARMED, podendo ser argumentado que serão as áreas sobre as quais as entidade administrativas e reguladores deverão orientar os seus esforços negociais de forma a tornar o processo de avaliação farmacoterapêutica e farmacoeconómica mais célere e eficiente para garantir uma utilização mais eficiente dos recursos financeiros do Estado disponíveis para a aquisição de medicamentos passíveis de constituírem reforço nos ganhos em saúde da população mas também em vantagem a médio-longo prazo no orçamento do Ministério da Saúde em Portugal. Segundo dados da European Federation of Pharmaceutical Industries and Associations (EFPIA), em 2011, dos 16000 medicamentos em desenvolvimento, 6317 correspondiam a medicamentos utilizados no tratamento de doenças oncológicas (correspondendo a cerca de 40\%), sendo a neurologia e as doenças do sistema nervoso central a segunda área terapêutica com maior número de medicamentos em desenvolvimento (2125, ou seja, 13\% do total) (EFPIA, 2014).

A decisão de financiamento por parte do Estado dos medicamentos no SNS, à luz dos princípios da governança, deverá garantir que o medicamento em causa traz verdadeiros ganhos em saúde à população à qual se destina, deverá visar a garantia da sustentabilidade e da utilização eficiente dos recursos do Estado, mas também deverá garantir o justo 


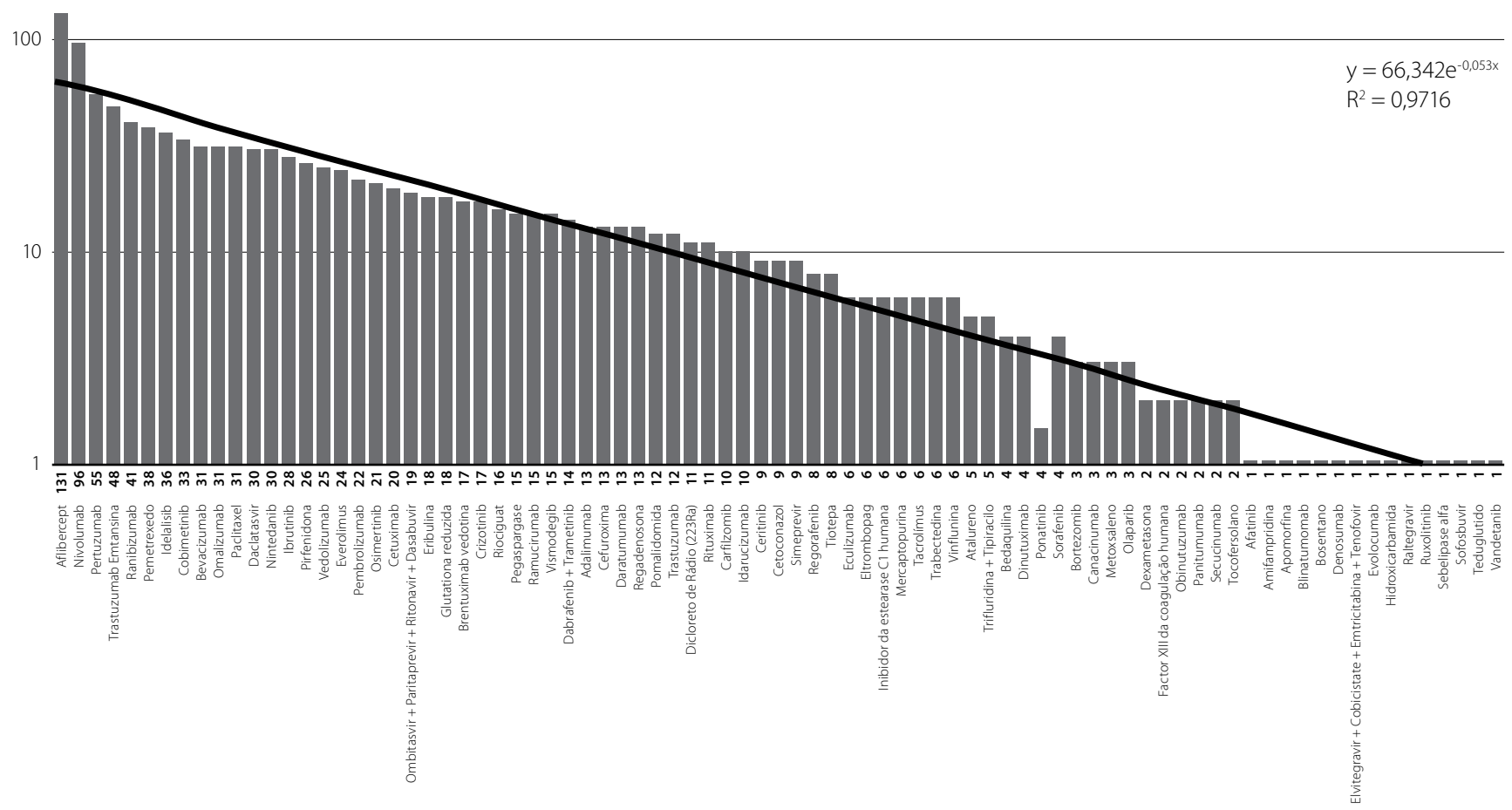

Fonte: elaboração própria com base em dados do INFARMED.

Figura 2. Frequência absoluta de cada medicamento, 1 de janeiro de 2016 a 31 de agosto de 2016 (escala logarítmica).

reconhecimento sob a forma de condições contratuais justas com a empresa detentora da AIM, tendo em consideração que, no panorama atual, a grande fatia do investimento em inovação provém de instituições privadas e que é dever do Estado garantir que a sua população tem acesso à inovação que Ihe garante ganhos reais nos cuidados de saúde. O custo estimado de introdução de um medicamento novo no mercado é de cerca de 1200 milhões de euros e o tempo médio de todo o desenvolvimento até à introdução no mercado ronda os 10 a 15 anos (EFPIA, 2015).

Após definição técnica da eficácia de determinado medicamento através dos programas de desenvolvimento clínico a que os medicamentos são sujeitos para que possam ser considerados para uso humano em determinada indicação, e após autorização de introdução no mercado desse medicamento em Portugal, o tempo que decorre entre a entrada de um processo de avaliação farmacoeconómica e farmacoterapêutica no INFARMED até à decisão ou não da comparticipação deverá ser o mais breve possível de forma a diminuir a necessidade de recorrer às AUE que poderão impactar negativamente o orçamento do Ministério da Saúde. Existe uma necessidade real de otimizar o processo de avaliação das tecnologias de saúde com vista à decisão do seu financiamento por parte do SNS.

Será interessante efetuar uma análise do real impacto económico das AUE tendo em consideração o tempo médio do processo de avaliação farmacoeconómica do INFARMED, em comparação com uma decisão atempada do financiamento dos medicamentos.

\section{Referências bibliográficas}

Baldwin, R., Scott, C., \& Hood, C. (1998). A Reader on Regulation. Oxford: Oxford University Press.

Cleveland, H. (1972). The Future Executive: A Guide for Tomorrow's Managers (1st ed.). New York: Harper \& Row.

Commission On Global Governance. (1995). Our global neighbourhood : the report of the Commission on Global Governance. Civil Wars. https://doi. org/GLOB CGG

Cook, P., Kirkpatrick, C., Minogue, M., \& Parker, D. (Eds.). (2005). Leading Issues in Competition, Regulation and Development. Cheltenham, UK and Northampton, USA: Edward Elgar.

Correia, P., \& Jesus, I. (2016). Combate às transferências bancárias ilegítimas pela Internet no direito português:entre as experiências domésticas e políticas globais concertadas. Revista Direito GV, 12(2), 542-563. https:// doi.org/10.1590/2317-6172201622

EFPIA (2015). The Pharmaceutical Industry in Figures - Key Data 2015. Brussels. Retrieved from https://efpia.eu/media/25822/2015-the-pharmaceuticalindustry-in-figures.pdf

EFPIA (2014). The Pharmaceutical Industry in Figures - Key Data 2014. Brussels. Retrieved from http://www.efpia.eu/uploads/Figures_2014_Final.pdf

Emmerij, L., Jolly, R., \& Weiss, T. G. (2001). Ahead of the Curve? UN Ideas and Global Challenges. Bloomington, IN: Indiana University Press.

Frederickson, H. G. (2009). Whatever Happened to Public Administration?: Governance, Governance Everywhere. In The Oxford Handbook of Public Management. https://doi.org/10.1093/ oxfordhb/9780199226443.003.0013

Frederickson, H. G., Smith, K. B., Larimer, C. W., \& Licari, M. J. (2012). The Public Administration Theory Primer. https://doi.org/10.5860/CHOICE.41-2423

INFARMED (2016). Deliberação n.o 662/2016 - Regulamento de funcionamento da Comissão de Avaliação de Tecnologias de Saúde CATS. Portugal: Diário da República. 
Jayasuriya, K. (2001). Globalization and the changing architecture of the state: the regulatory state and the politics of negative coordination. Journal of European Public Policy, 8(1), 101-123. https://doi. org/10.1080/1350176001001859

Jordana, J., \& Levi-Faur, D. (2003). The Rise of the Regulatory State in Latin America: A Study of the Diffusion of Regulatory Reforms across Countries and Sectors. In Paper presented at the Annual Meeting of the American Political Science Association. Philadephia.

Jordana, J., \& Levi-Faur, D. (2004). The Politics of Regulation: Institutions and Regulatory Reforms for the Age of Governance. (J. Jordana \& D. LeviFaur, Eds.). Cheltenham, UK: Edward Elgar Pub.

Lynn Jr, L., \& Heinrich, C. (2000). Governance and Performance: New Perspectives. Washington, DC: Georgetown University Press.

Lynn Jr, L., Heinrich, C., \& Hill, C. (1999). The Empirical Study of Governance: Theories, Models, Methods. In Workshop on Models and Methods for the Empirical Study of Governance. Tucson, AZ: University of Arizona.

Lynn Jr, L., Heinrich, C., \& Hill, C. (2001). Improving Governance: A New Logic for Empirical Research Improving Governance: A New Logic for Empirical Research (American Government and Public Policy). Washington, DC: Georgetown University Press.

Majone, G. (1994). The rise of the regulatory state in Europe. West European Politics, 17(3), 77-101. https://doi.org/10.1080/01402389408425031

Manzetti, L. (2000). Regulatory Policy in Latin America: Post-privatization Realities. Miami: North-South Center Press.
Ministério da Saúde (2006). Decreto-lei no 176/2006, de 30 de agosto Regime jurídico dos medicamentos de uso humano, Pub. L. No. arto 920. Portugal: Diário da República.

Ministério da Saúde (2012). Decreto-Lei No 46/2012, de 24 de fevereiro. Portugal: Diário da República.

Ministério da Saúde (2015). Decreto-Lei no 97/2015, de 1 de junho. Portugal: Diário da República.

Nanda, V. (2006). The "Good Governance" Concept Revisited. Annals of the American Academy of Political and Social Science, 603 (Law, Society, and Democracy: Comparative Perspectives), 269-283. https://doi. org/10.2307/25097772

Newman, J. (2000). Modernizing Governance: New Labour, Policy and Society. Thousand Oaks, CA: SAGE Publications Ltd.

Stigler, G. J. (1975). Citizens and the State: Essays on Regulation. Chicago, IL: University of Chicago Press.

Vogel, S. (1998). Freer Markets, More Rules: Regulatory Reforms in Advanced Industrial Countries (Cornell Studies in Political Economy). Ithaca and London: Cornell University Press.

Weiss, T. G. (2000). Governance, Good Governance and Global Governance: Conceptual and Actual Challenges. Third World Quarterly, 21(5), 795-814. https://doi.org/10.1080/713701075 